\title{
DIE ERSTELLUNG EINES COMICS ALS LERNMEDIUM FÜRLESEKOMPETENZ IM DEUTSCHUNTERRICHT
}

\author{
Berlian Putri Sutrisno Nanico \\ Ahmad Bengar Harahap \\ Hafniati
}

\begin{abstract}
AUSZUG
Das Ziel dieser Untersuchung ist es, um eines Comics als Lernmedium für Lesekompetenz im Deutschunterricht zu erstellen. In dieser Untersuchung wurden die Lernmaterialien zum Thema Alltags leben Kennenlernen und im Cafe erstellt. Die Datenquelle dieser Untersuchung ist das Buch Studio d A1Der Prozess besteht aus der Erklärung die Schritte von ADDIE Model , Die Schrittebesteht aus:(1) Analyse, (2) Design,(3) Entwicklung, (4) Durchführung und (5).Bewertung.Lernmedium mit demApplication Comidea app erstellt wurden,können in den Lernprozessunterstützen und das Verständnis der deutschen Sprache. Die Qualität des Comics basierend auf der Note.
\end{abstract}

Schlüsselwörter :Comics, Lernmedium.

\section{EINLEITUNG}

Heute haben die Deutschlernende weniger Interesse am Lesen. Die Deutschlernende lesen in der Regel nur Texte, unattraktiv und langweilig. Dass die Deutschlernende beim Schwieirigkeiten haben, wird an nicht befriedigenden Testergebnissen deutslich. Diese kommen zustande, weil die Schüler nicht verstehen, was sie lesen. Leseverständnis ist eine wichtige Tätigkeit, um Wissen und Informationen zu erwerben. In den Schulen verwenden die Lehrer meist den Vortrag als Lehrmethode. Die Deutschlernende benötigen ein Medium zur Unterstützung, um ihre Lesefähigkeiten zu verbessern und den Inhalt gelesener Text zu verstehen. Deshalb entwickelt die Verfaserin ein Lernmedium in Form eines Comic, das dazu dienen soll, den Schüler das Leseverständnis zu erleichtern.

Lernmedium mit dem Application Comidea erstellt wurden, können in den Lernprozess unterstützen und das Verständnis der deutschen Sprache. Comidea ist eine Comic-APP über die Sie Ihre Comic-Werke erstellen und teilen können. Es ist einfacher zu bedienen und keine Notwendigkeit für irgendeine komische elementare. Die Art von Comics erstellt ist eine Mischung aus Comic Bucher und Comic Wissen . Die Daten von dieser Untersuchung ist die Redemiteln mit Thema Altags leben (Kennenlernen, und im Cafe) von Buch Studio d A1. Das Lesen der Comic selbst ist 
vor allem bei Deutschlernende sehr beliebt, weil der Inhalt meist lustig ist und die Geschichte nicht nur Text benutzt sondern auch durch viele interessante Bilder erzählt wird (Daryanto, 2010 : 128). Die Rolle der Comics als Lernmedium kann Phantasie und kreatives Denken fördern. Durch ein solches Lernmedium können die Deutschlernende die zu lesen den Texte mit Hilfe von Visualisierung einfacher lesen und verstehen.

Vom kognitiven Aspekt her kann ein Bild Menschen dabei helfen, sich leichter an Informationen zu erinnern. Das Ergebnis sagt aus einer Studie, dass Kinder, die eine Geschichte mit Bildern lesen, eine bessere Leistung liefern können, da durch Bilder das Verständnis verbessert werden kann.

\section{THEORETISCHE GRUNDLAGE}

\section{Das Ziel die Validität}

Die Validierung das Comics als Lernmedium besteht aus zwei Teilen.Zuerst ist die Evaluation der Wörter und der Sätze, die von dem Muttersprachler gemacht wird. Danach wird das Design evaluiert, das von Experten des Designs gemacht wird.

\section{Die Reliabilität}

Lernmedium mit dem Application Comidea erstellt wurden, können in den Lernprozess unterstützen und das Verständnis der deutschen Sprache.Neben verbessern wurde als Innovation gemacht Schüler Interesse am Lesen und Verstehen zu erhöhen. Comicsinteressant gestaltet und angepasst an die benötigten Materialien Schüler.Diese Untersuchung verwendet ADDIE Modell.ADDIE Theorie (AnalyseDesign-Entwicklung-Durchführung-Bewertung). Die Schritte, die im ADDIE Modell zeigen, besteht aus: (1) Analyse, (2) Design, (3) Entwicklung, (4) Durchführung und (5) Bewertung.Der Autor verwendet die Anwendung Comidea Program Comics in der Gestaltung. Schritte, um Comics Comidea zu machen;. Werkzeuge und grundlegende Charakterbildung ,Brain-Storming, Einrichtung einer Parzelle, Storyboard und Naming, Täfelung,Spezialeffekt, und Design Cover.

\section{Technik der Analyse die Qualität}


https://jurnal.unimed.ac.id/2012/index.php/studia/index

Auf dieser Stufe wurden der Lernmedien Comics Bewertung gemacht. Diesen Schritt wird Ratschläge und Anregungen erwarten, die Qualität der Lehre Medien verbessern. Diese Experten sind deutsche Muttersprachler und Experten des Design. Die Qualität des Comics basierend auf der Note. sen werden,damit die sprachlichen Fehler verbessert werden können.

\section{DIE DATENBESCHREIBUNG}

ieses Kapitel handelt von den Untersuchungsergebnissen. In diesem Kapitel werden der Prozess und die Erstellung Eines Comics Als Lernmedium Für Lesekompetenz Im Deutschunterricht. Die Schritte und die Ergebnisse der Untersuchung werden in diesem Kapitel erklärt

\section{Die Validität des Testitems testen}

Auf dieser Stufe wurden der Lernmedien Comics Bewertung gemacht. Diesen Schritt wird Ratschläge und Anregungen erwarten, die Qualität der Lehre Medien verbessern. Diese Experten sind deutsche Muttersprachler und Experten des Design. Die Qualität des Comics basierend auf der Note. Die einer Expertin gegeben hat, ist gut mit der Note 97,5 (sehr gut).Die Kommentare einer Expertin an des Comics ist, dass die Comic ist innovativ, gut erstellt, interessant und sehr motivierend. Es ist sehr ratsam,die Texte im Comic von einem deutschen Muttersprachler noch einmal gelesen werden,damit die sprachlichen Fehler verbessert werden können.

Die Ergebnisse des Designs das Comics als Lernmedium für lesekompetenz

\section{im Deutschunterricht}

Die Einbände des Lernmediums 

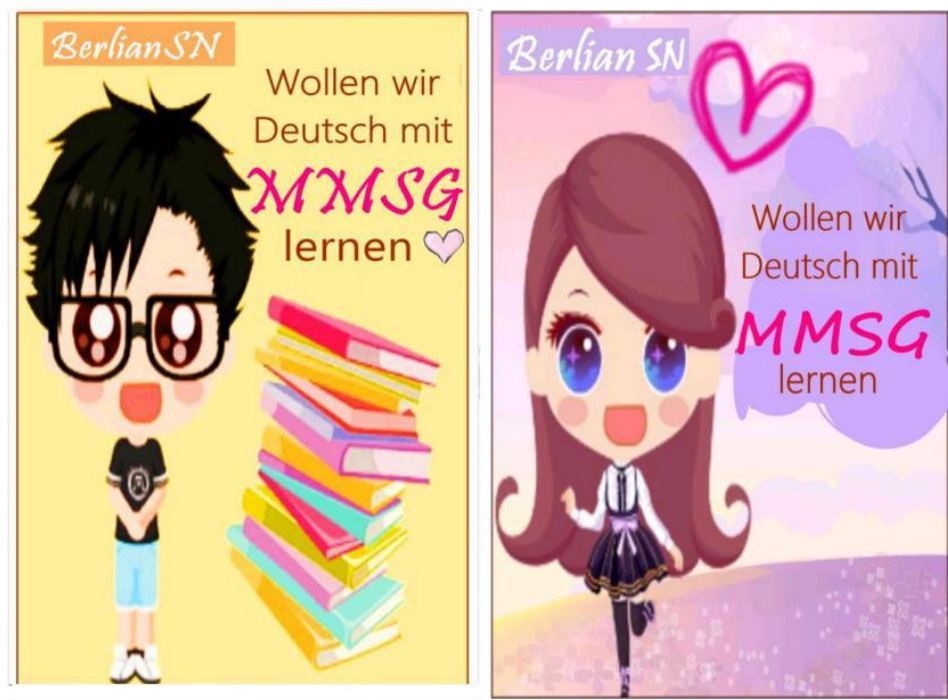

Ein Beispiel dem Ergebnis für das Comic kennenlernen
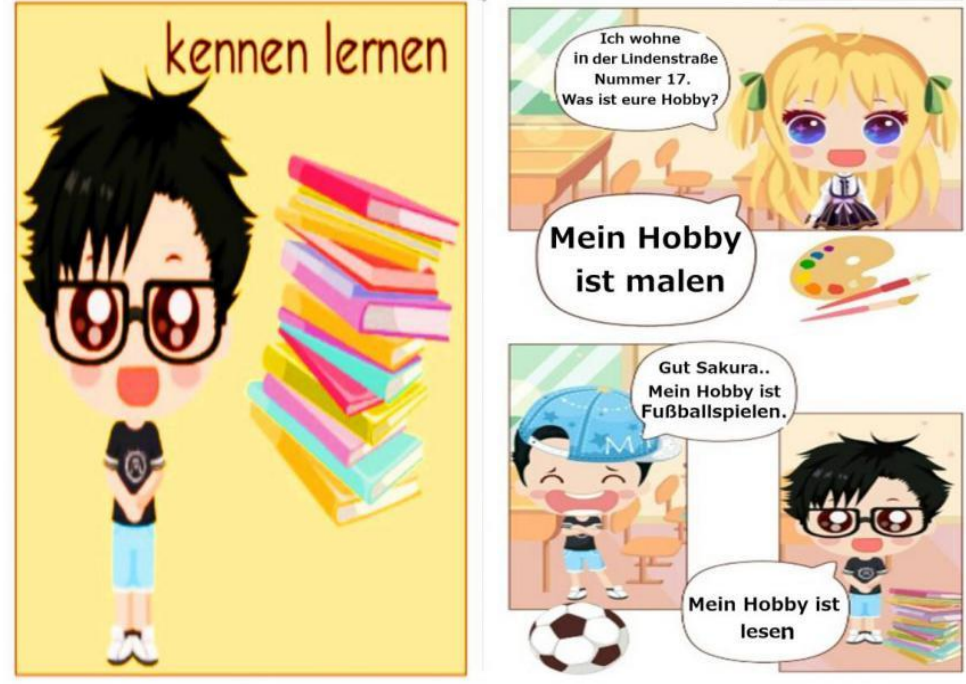

Ein Beispiel dem Ergebnis für das Comic im Café 


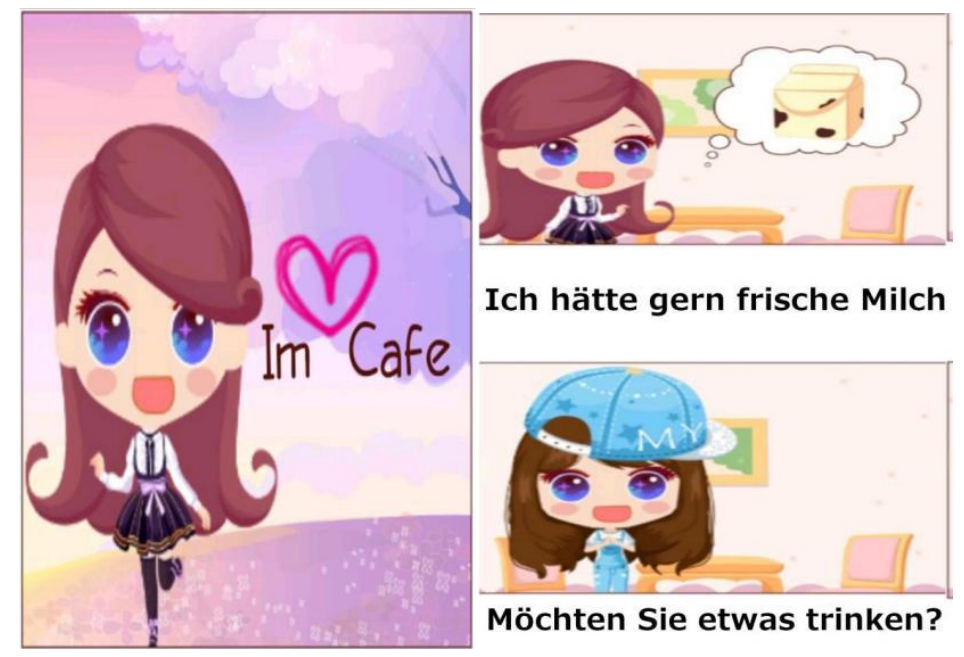

Die Diskussion

Basierend auf den Ergebnisse einesComics als Lernmedium für die lesekompetenz im Deutschunterricht wurde zusammengefasst, dass die Daten in dieser Untersuchung Redemittel aus dem Buch "Studio d A1" In dieser Untersuchungs wird die ADDIE Theori benutzt. Diese Theori besteht aus fünf Phase. Die Phasen sind (1) Analyse, (2) Design, (3) Entwicklung, (4) Durchführung und (5) Bewertung.. In diesem Kapitel wird jede Stufe mit ihrem Ergebnis erklärt. In dieser Phase der Beobachtung werden Informationen über das Lernen der Deutschlernenden, ihrer Bedürfnisse und die Lernmedien im Deutschunterricht gesammelt. Diezweite Phase ist Design. Zu diesem Zeitpunkt entwarfen die Autoren eine Comic-Story und die Lernthemen anzupassen. Der Autor verwendet die Anwendung Comidea Program Comics in der Gestaltung. Schritte, um Comics Comidea zu machen,Tools und grundlegende Charakterbildung,Brain-Storming, Einrichtung einer Parzelle, Storyboard und Naming, Täfelung,Spezialeffekt und Design Cover. Die nächste Phase ist die Entwicklung.Diese Phase ist die Vorbereitung aller Mittel und Materialien, die für die Erstellung der Lernmedien Comicss verwendet werden sollen, nämlich: die Computer / Handy mit Comiidea Anwendung. Dann erstellt man das 
Lernmedium das Comics mit ThemaAltags leben (Kennenlernen ,Essen und Trinken).Danach ist Durchführung. Die Umsetzung ist ein konkreter Schritt ein Lernsystem zu implementieren, das erstellt wird. Das heißt, in dieser Phase alles das entwickelt wurde, installiert ist oder in einer solchen Art und Weise angebracht, die Rollen oder Funktionen eingestellt umgesetzt werden.

Die Validierung das Comics als Lernmedium besteht aus zwei Teilen.Zuerst ist die Evaluation der Wörter und der Sätze, die von dem Muttersprachler gemacht wird. Danach wird das Design evaluiert, das von Experten des Designs gemacht wird. Danach wird in der Revisionsphase schon das Comics als Lernmedium erstellt. Von 10 Kategorien der Aspekt der Evaluation gibt die Expertin die Note vier für die neun Aspekt und 3 für die ein Aspekt (siehe Anhang V).

Basierend auf die obigen Erklärung kann zusammengefasst werden, dass die Erstellung eines Comics als Lernmedium für lesekompetenz im Deutschunterricht zum Thema ,alltags leben” aus dem Buch Studio d A1"mit der ADDIE Model sehr gut ist. Sodass die Dozenten oder die Studenten als das Lernmedium benutzen kann.

\section{SCHLUSSFOLGERUNG}

Nach den Untersuchungsergebnissen fallen die Schlussfolgerungenfolgendermaßen aus:

1. Der Prozess der Erstellung eine Comics als Lernmedium für Lesekompetenz im Deutschunterricht besteht aus der Erklärung die Schritte von ADDIE Teorien, Die Schritte besteht aus: (1) Analyse, (2) Design,(3) Entwicklung, (4) Durchführung und (5) Bewertung.

2. Das Ergebnis der Erstellung einesComics als Lernmedium für Lesekompetenz im Deutschunterricht sind:

a. In dieserUntersuchung hat einesComics als Lernmedium für lesekompetenz im Deutschunterricht erstellt,und das Produk wird in diese Abschlussarbeit angehängt. 
b. Die Erstellung einesComics als Lernmedium für lesekompetenz im Deutschunterricht haben von Experten evaluiert. Die Validierung das Comics als Lernmedium besteht aus zwei Teilen.Zuerst ist die Evaluation der Wörter und der Sätze, die von dem Muttersprachler gemacht wird. Danach wird das Design evaluiert, das von Experten des Designs gemacht wird. Basierend auf der obigen Meinung kann geschlossen werden, dass dieses Comics mit Comiidea appalsLernmedium beim Deutschunterricht.Als Erfindung, die alle Deutschlernende können dieses Comics mit modernen elektrischen Geräte wie PC, Händy, Android oder "Smartphone" heuzutage beim lernen passen des herunterladen.

\section{LITERATURVERZEICHNIS}

Arsyad, Azhar. 2009. Media Pembelajaran.Jakarta : PT Raja Grafindo Persada.

Azies, Furqanul dan Alwasilah, A Chaedar. 1996.Pengajaran Bahasa Komunikatif: Teori danPraktek. Bandung: PT Remaja Rosdakarya.

Barus, Ulian dan Suratno. 2015. Pemanfaatan Candi Bahal sebagai Media Pembelajaran Alam Terbuka Dalam Proses Belajar Mengajar. Medan: Perdana Mitra Handalan

Bausch, et al. 2007. Handbuch Fremdsprachenunterricht. Tübingen: Narr Francke

Choiriyatuna'im Amaliya. 2014. Die Entwicklung Des Comics “Die Geschichte der Familie Gudrun Deutsch im Alltag " als unterrichtsmedien fur Lesevestandis der Schuler Klasse XI SMA.Universitas Negeri surabaya. Jurnal.

D.Meidany,Cindy .2015. Step By Step Belajar Bahasa Jerman. Klaten :Caesar Media Pustaka.

Funk, Hermann. 2013. Studio d Al Deutsch als Fremsprache. Jakarta :Katalis

Maeyama Machiko. 2008. Ayo Bikin Komik dan Mewarnai.Jakarta :PT Elex Media Komputindo.

Osa Amanokawa. 2007.Guide to Draw Manga vol4; Menggambar Komik.Yogyakarta: C.V Andi Offset.

Rozalena,Agustin dan Dewi, Sri komala. 2016. Panduan Praktis Menyusun Pengembangan Karier dan Pelatihan Kariawan. Jakarta: Raih Asa Sukses.

Susilana, Rudi dan Riyana, Cepi. 2009. Media Pembelajaran. Bandung: CV Wacana Prima 
Studia: Journal des Deutschsprogramms p-ISSN 2301-6108 e-ISSN 2654-9573

https://jurnal.unimed.ac.id/2012/index.php/studia/index

http://www.ninthart.com/german/, (diaksespada 1 )gelessen am 1April 2017, um $11.00 \mathrm{uhr}$

http://appbalo.com/apps/comidea-writing-comics-app/,gelessen am 1juni 2017, um $14.00 \mathrm{uhr}$

http://a/pengembanganaddie/ModelPembelajaran/ADDIE\%20/,gelessen am 3juni 2017, um $08.00 \mathrm{uhr}$

Berlian Putri Sutrisno Nanico is an alumni of German language study program of Unimed, Medan 
Studia: Journal des Deutschsprogramms p-ISSN 2301-6108 e-ISSN 2654-9573

https://jurnal.unimed.ac.id/2012/index.php/studia/index 